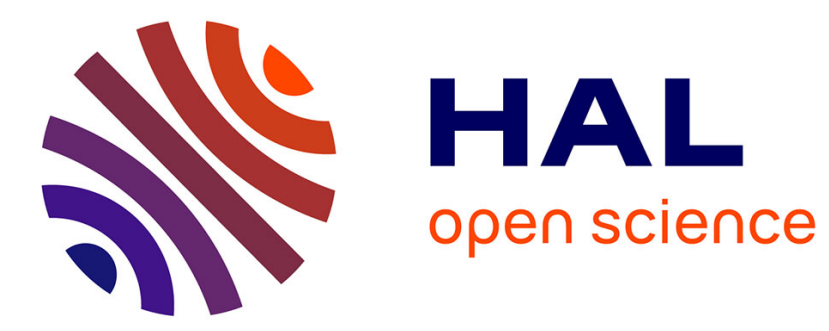

\title{
Automatic Localization of Gas Pipes from GPR Imagery
}

\author{
Guillaume Terrasse, Jean-Marie Nicolas, Emmanuel Trouvé, Émeline Drouet
}

\section{To cite this version:}

Guillaume Terrasse, Jean-Marie Nicolas, Emmanuel Trouvé, Émeline Drouet. Automatic Localization of Gas Pipes from GPR Imagery. EUSIPCO, Aug 2016, Budapest, Hungary. hal-01351239

\section{HAL Id: hal-01351239 \\ https://hal.science/hal-01351239}

Submitted on 3 Aug 2016

HAL is a multi-disciplinary open access archive for the deposit and dissemination of scientific research documents, whether they are published or not. The documents may come from teaching and research institutions in France or abroad, or from public or private research centers.
L'archive ouverte pluridisciplinaire HAL, est destinée au dépôt et à la diffusion de documents scientifiques de niveau recherche, publiés ou non, émanant des établissements d'enseignement et de recherche français ou étrangers, des laboratoires publics ou privés. 


\title{
Automatic Localization of Gas Pipes from GPR Imagery
}

\author{
Guillaume Terrasse*, Jean-Marie Nicolas*, Emmanuel Trouvé ${ }^{\dagger}$ and Émeline Drouet $^{\ddagger}$ \\ * LTCI, CNRS, Télécom ParisTech, Université Paris-Saclay, 75013, Paris, France. \\ $\dagger$ LISTIC, Polytech Annecy Chambéry, Université Savoie Mont-Blanc, 74944, Annecy-le-Vieux, France. \\ $\ddagger$ CRIGEN, ENGIE, Direction Recherche et Innovation, 93211, Saint-Denis La Plaine, France. \\ (corresponding author, e-mail : guillaume.terrasse@ telecom-paristech.fr)
}

\begin{abstract}
In order to improve asset knowledge and avoid third part damages during road works, the localization of gas pipes in a non-destructive way has become a wide domain of research during these last years. Several devices have been developed in order to answer this problem. Acoustic, electromagnetic or RFID technologies are used to find pipes in the underground. Ground Penetrating Radar (GPR) is also used to detect buried gas pipes. However it does not directly provide a $3 D$ position but a reflection map called B-scan that the user must interpret. In this paper, we propose a novel method to automatically get the position of gas pipes with GPR acquisitions. This method uses a dictionary of theoretical pipe signatures. The correlation between each atom from the dictionary and the B-scan is used as feature in a two part supervised learning scheme. Our method has been applied to real data acquired on a test area and in real condition. The proposed method presents satisfying qualitative and quantitative results compared to other methods.
\end{abstract}

Index Terms-Gas pipes localization, GPR, Dictionary, Supervised learning.

\section{INTRODUCTION}

While roadworks and civil engineering works operate near network pipes, an inaccurate mapping of pipe networks can cause damages which lead to human and economical damages. In France, 156 damages on gas pipelines have been counted during the 2012 year [1]. Precise location of buried gas networks in a non-destructive manner becomes primordial for all network system operators. Several technologies have been developed to update maps of underground network or to localize pipes in real time before works which could affect them. For this purpose, some technologies use the propagation of acoustic or electromagnetic waves in a passive or active mode. Another technology uses an RFID chip placed on the pipe to read information (3D position and characteristics).

In our work, we are interested in using the Ground Penetrating Radar (GPR) technology to get the position of the gas pipes. However this sensor does not provide a position in the three dimensions but a reflection map called B-scan which offers a high degree of freedom in result interpretation. Operators need experience to understand this particular data. In order to help the non-expert users of GPR to detect and find the position of buried pipes, a novel method to automatically localize them in the B-scan has been developed. The ground penetrating radar has been widely used in different applications like civil engineering, geological study or glaciology [2].
This device is equipped with a transmitting and receiving antenna with a large band and a high frequency (from 100 $\mathrm{MHz}$ to $2 \mathrm{GHz}$ ) placed few centimetres above soil surface. An electromagnetic wave is sent in the underground which is backscattered when it encounters a heterogeneity. The shape of the impulse signal looks like a Ricker wavelet (Fig 1 (a)). Then the receiving antenna records the backscattered wave at each position as function of time. This function is called an Ascan. By moving the GPR, a collection of A-scans is recorded at different positions known thanks to an odometer (Fig 1 (b)). This ensemble of A-scans forms a B-scan in which each value corresponds to the amplitude of the backscattered wave at a certain position of recording and time (Fig 1 (c)). On a Bscan, the shape of the ensemble of echoes and their intensity inform about the nature of the object. Thereby, an hyperbolic shape means there is a punctual object, for instance a pipe or a rock. On the other hand, a linear shape could be a boundary between two layers.

Therefore in order to localize the pipes, we have to detect hyperbolas in the B-scan. The automatic detection of hyperbolas offers several advantages :

Easier interpretation : Indeed, the GPR records a lot of information from the soil which makes reading the B-scan hard for a non-expert operator. The overprint of an hyperbola on the B-scan can help him to better understand the data and to work faster.

Time/depth conversion : After scanning the underground, a $3 \mathrm{D}$ position is not directly obtained but a $2 \mathrm{D}$ position according to the position of the GPR and a back and forth time travel of the wave. In order to get a depth scale, the wave speed must be estimated in the soil. This can be done with the shape of the hyperbola.

Whole pipe 3D position : Indeed the hyperbola detection in each B-scan in a sequence of acquisitions along a pipe allows the false alarm removal thanks to the redundant information, a faster update of maps and a merging data with other devices (acoustic, RFID, electromagnetic).

Several studies addressed the automatic detection issue in B-scans. Most of them used supervised learning algorithms. Thereby, in [3] the authors applied the algorithm Viola Jones. This algorithm uses Haar wavelet to compute the feature in input of a boosting learning algorithm, Adaboost. 


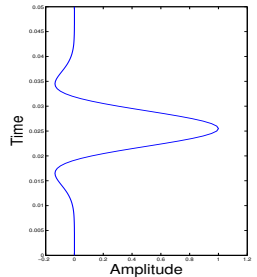

(a) Ricker wavelet

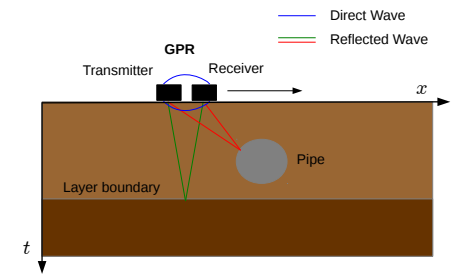

(b) Auscultation of the soil

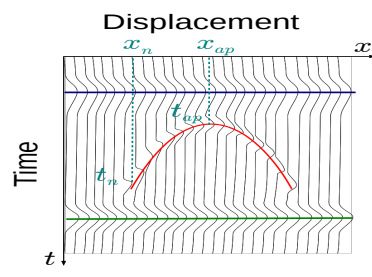

(c) Ensemble of A-scans

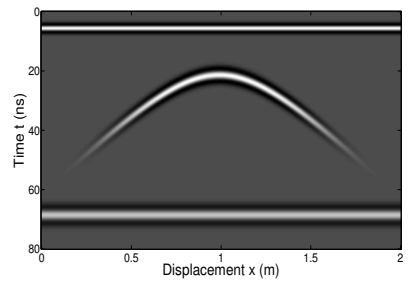

(d) Simulated B-scan

Figure 1: Building of a B-scan

The authors of [4] and [5] used Neural Network. In particular, in [4], they proposed to use trapezoidal patches to minimize the amount of background in patches with hyperbola during the learning step. We call patch, a local representation of an image, namely a small group of close pixels. In [6], the authors proposed a classification method from A-scan. For this, they used an adaptive dictionary of 1D gabor wavelets to compute a sparse decomposition of the signal. Then they applied the parameters used during the sparse decompositon of the A-scan as feature in a classification step with the Suport Vector Machine (SVM). For the detection results of these works, the algorithm proposed by [3] shows a detection rate from $59 \%$ to $76 \%$ on three different test sets. The method of the authors of [6] obtained a detection rate between $91.7 \%$ and $99.3 \%$ according to the dataset. However these last results have been obtained from the training set and so they cannot be compared with results obtained from a test set independent of the learning step. [4] and [5] did not mention their detection rates. In order to denoise seismic data a new frame with hyperbolic shape has been developed by [7]. The frame is built on three parameters. Both parameters jointly modified the thickness and shape of hyperbola. The last one influence the flattening of the hyperbola top. These parameters also act on the position of the hyperbola top. Our work has been inspired by the frame of [7] to build a dictionary of theoretical pipe signatures. Nevertheless, in our case, the atoms remain centred regardless the parameter values. The thickness, the shape and the flattening of the atom are controlled by independent parameters.

In the subsequent part, the proposed method is presented. Especially the dictionary and the supervised learning scheme are detailed. Then, qualitative and quantitative results are shown from real data and compared with other methods.

\section{Proposed Method}

The correlation between the atoms of the dictionary and the B-scans is used as feature in input of a supervised learning scheme. First of all the dictionary and the feature coefficients computation are described. Then the supervised learning steps for the detection of hyperbola are detailed.

\section{A. Dictionary}

The dictionary is composed of hyperbolas $(g(x))$ convolved with a Ricker wavelet $(r(t))$ according to vertical axis.
Each atom is parametrized by $\Theta=\left\{\alpha, \sigma, f, a, x_{0}, t_{0}\right\}$ We define an atom $h(x, t \mid \Theta)$ of our dictionary :

$$
\begin{aligned}
h(x, t \mid \Theta) & =e^{-\alpha \cdot A(x, t)} \cdot(r(t) \star \delta(t-g(x))) \\
r(t \mid \sigma) & =\frac{2}{\sqrt{3 \sigma} \cdot \pi^{1 / 4}} \cdot\left(1-\frac{t^{2}}{\sigma^{2}}\right) \cdot e^{-\frac{t^{2}}{2 \sigma^{2}}} \\
g\left(x \mid x_{0}, t_{0}, a, f\right) & =a \cdot \sqrt{f^{2}+\left(x-x_{0}\right)^{2}}+t_{0}-f . a
\end{aligned}
$$

where $\delta($.$) is the Dirac distribution. \alpha$ and function $A(x, t)$ impact the atom attenuation. Especially, as we are working in a finite space, $A$ ensures a low energy of the atom close to the boundaries. $\sigma$ parameter is the standard-deviation of the Ricker wavelet and controls the thickness of the atom. Parameter $a$ affects the opening of hyperbolas (spacing of the hyperbola branches) which is directly related to the wave velocity in the soil. Then $f$ also affects the shape of the hyperbola but plays a role in the flattening of the top of hyperbolas located at $\left(x_{0}, t_{0}\right)$.

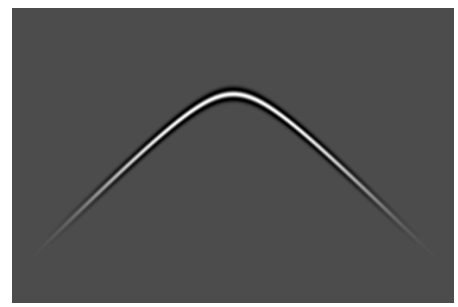

(a) $f=50 ; a=1.5 ; \sigma=5$

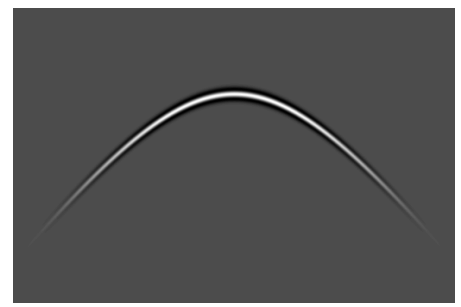

(b) $f=150 ; a=2 ; \sigma=5$
Figure 2: Atom examples with different parameters

Because the hyperbola shape mainly depends on the parameter $a$, in practice to keep a reasonable dictionary size, $\alpha, f$ and $\sigma$ are set such that only one shape parameter and two position parameters remain. The coefficient $\mathrm{C}$ is computed according to the parameters, in our case just $a, x_{0}$ and $t_{0}$ vary $\left(\Theta=\left(a, x_{0}, t_{0}\right)\right)$ :

$$
C\left(x, t \mid a, x_{0}, t_{0}\right)=\sum_{x=1}^{X} \sum_{t=1}^{T} Y(x, t) \cdot h\left(x, t \mid a, x_{0}, t_{0}\right)
$$

where $Y$ is a B-scan of dimension $X \times T$.

With an atom centred, it amounts to :

$$
C\left(x, t \mid a, x_{0}, t_{0}\right)=\sum_{x=1}^{X} \sum_{t=1}^{T} Y(x, t) \cdot h\left(x-x_{0}, t-t_{0} \mid a\right)
$$




$$
\begin{aligned}
C\left(x, t \mid a, x_{0}, t_{0}\right) & =\left(Y \star \overline{h_{a}}\right)\left(x_{0}, t_{0}\right) \\
\text { where } \overline{h_{a}} & =h(-x,-t \mid a)
\end{aligned}
$$

The parameters $x_{0}$ and $t_{0}$ are also set such that each atom are centred. Therefore, the correlation between the B-scan to analyse and each atom is computed and an image stack is obtained (Fig : 5 (1)). Each image from the stack is the correlation of B-scan and an atom (Figure 4 (b)). Thus a pixel from the B-scan is associated with a vector of coefficients $\mathbf{C}_{x, t}=\left(C\left(x, t \mid \Theta_{1}\right), \ldots, C\left(x, t \mid \Theta_{K}\right)\right)^{T}$, with $K$, the number of atoms in the dictionary. We can see $\mathbf{C}_{x, t}$ as a local score of the correlation between the B-scan and each atom.

High value coefficients would show the position of an hyperbola. Thus a simple way to detect hyperbola would be to keep these coefficients by a thresholding step. However high value coefficients do not always correspond to the presence of an hyperbola top and they lead to an important false alarm rate. Figure 3 illustrates the computation of correlation between an atom (green) and an horizontal clutter with high energy (dark grey) and a background with a low energy (light grey). Thus, the correlation value corresponds to the contribution of the inner product between the part of the atom which intersects the clutter (red hatched), the one which intersects the background and the B-scan. For an hyperbola with a total energy slightly above the background one its coefficient value will be lower than the clutter one.

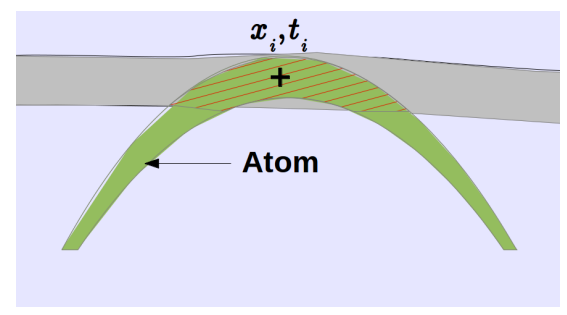

Figure 3: High value coefficient for clutter; Dark grey : clutter with high energy; Light gray : background with low energy; Red hatched area : Atom centred at $\left(x_{i}, t_{i}\right)$ intersecting clutter

Before starting the coefficient computation, in order to enhance hyperbola energy the B-scans are preprocessed to remove the clutter which could hide hyperbolas. We applied an algorithm based on curvelet transform [8] and an additional automatic gain which suppresses the main horizontal information in the B-scan, namely the direct wave and boundaries between layers.

Figure 4 (a) shows the original B-scan which has been acquired above an area with five pipelines. It seems easy to distinguish three of them but two hyperbolas (in the centre) are very hard to distinguish. Figure 4 (b) shows the coefficients $C\left(. \mid \Theta_{i}\right)$, where the atom parametrized by $\Theta_{i}$ is the most correlated with the hyperbolas in the B-scan. We noticed that the hyperbola energy is concentrated on the hyperbola top localization.

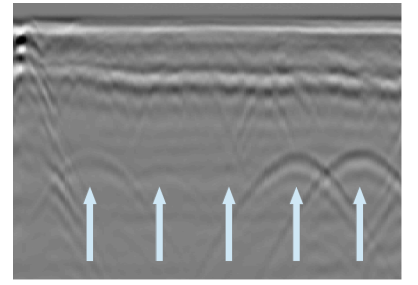

(a) Original B-scan

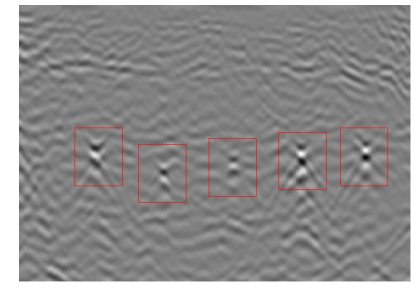

(b) Coefficients
Figure 4: Coefficients from image stack associated to the most correlated atom with the hyperbolas in the B-scan; Five blobs are shown in the red boxes (b)

Our problem is no longer to find hyperbolas but to detect blobs, marked in the red boxes in Figure 4 (b). A threshold would still lead to a too high false alarm rate. Hence, to detect these blobs in a robust manner, we apply a supervised learning algorithm.

\section{B. Supervised Learning}

The purpose of this part is to predict if there is an hyperbola top or not at a position $(x, t)$. The supervised learning part has been split into two models. The first model uses the coefficient vectors $\mathbf{C}_{x, t}$ whereas the second one uses blob patches. This allows us to be more robust. Indeed hyperbolas will be detected in two separated models. Moreover the blob detection from a patch search in the image stack is too computer intensive. A detection step is realized among $X \times T$ (B-scan size) coefficient vectors of $K$ values (the number of different hyperbolas in the dictionary, around 50) each, whereas with patches (size 40x40) it is done in the image stack namely $X \times T \times K$ patches have to be processed. Hence the first step which is lighter in computer resource greatly reduces the possibility number for the blob search.

Two models have been learnt with a supervised learning algorithm. The first one, the Dictionary model separates the coefficient vectors associated to a blob and those associated to the background (namely without blobs). The second one, the Blob model predicts if a patch contains a blob or not. For this purpose, a first database has been built with a set of coefficient vectors which are related to blobs and a set of coefficient vectors randomly chosen among a set of vectors without blob. The second database has been build with blob patches and background patches. The hyperbola detection method consists as follows (Fig 5) :

1) A coefficient vector for each position of the B-scan is computed $\mathbf{C}_{x, t}$.

2) Each coefficient vector is used in the Dictionary Model.

3) According to the classification results, coefficient vectors related to a position is kept or rejected.

4) From the kept position, we look at the atom the more correlated namely $j=\arg \max _{i}\left(C\left(x, t \mid \Theta_{i}\right)\right)$.

5) The Blob Model decides if a patch centred at $(x, t)$ at the $\mathrm{j}^{\text {th }}$ image from the image stack is a blob patch or not. If this the case then the position is kept as being an hyperbola top. 


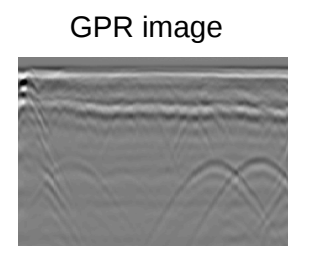

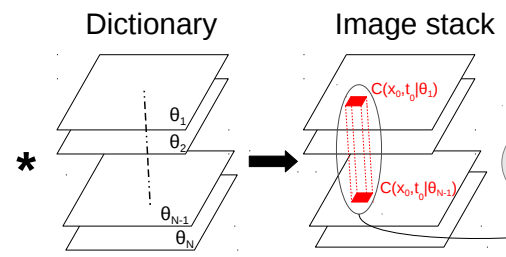

1

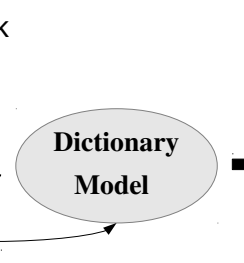

2

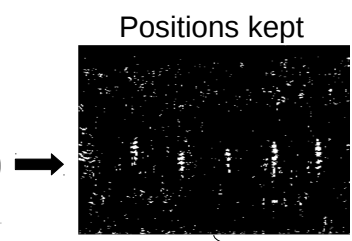

3

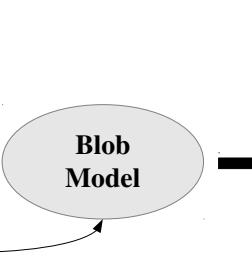

4
Positions kept

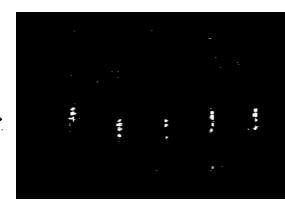

5

Figure 5: The proposed framework; 3 and $\mathbf{5}$ respectively show the positions kept in white after the binary classification by the models learnt based on coefficient vectors (Dictionary Model) and blob patches (Blob Model).

There are several algorithms for supervised learning, we chose to apply the Support Vector Machine (SVM) algorithm [9] with a RBF kernel for both learning steps. Figure 5 (3 and 5) presents the results of the two parts of the supervised learning step.

\section{Wave speed}

Once an hyperbola has been found its mathematical function is automatically given by the atom which is the more correlated with. Hence the averaged wave speed in the soil can be estimated.

Let $v$, the averaged wave speed in the underground, $d_{n}$ the distance between the pipe and the antenna at $n^{t h}$ emitted pulse at the position $x_{n}$ and $t_{n}$ the back and forth time of the wave after reflection on the pipe. $x_{a p}$ and $t_{a p}$ are respectively, the GPR device position and back and forth wave time when the GPR is located above the hyperbola apex (Fig 1 (c)).

$$
v=2 \sqrt{\frac{\left(x_{n}-x_{a p}\right)^{2}}{t_{n}^{2}-t_{a p}^{2}}}
$$

Hence with the automatic hyperbola detection with our dictionary, we can convert the time scale in a depth scale and finally we can get an estimated depth.

\section{RESULTS}

In order to assess the performances of the proposed method, a set of B-scans has been acquired by a $500 \mathrm{MHz}$ antenna USRADAR GPR and a $400 \mathrm{MHz}$ antenna GSSI GPR on a test area where the nature of the soil, the $3 \mathrm{D}$ position, diameter (from $20 \mathrm{~mm}$ to $160 \mathrm{~mm}$ ) and the material (polyethylene, cast iron, steel) of pipes are known. This configuration diversity allows us to observe a lot of different responses from pipes relative to the soil.

Figure 6 illustrates the final result of the hyperbola detection and shape estimation from two preprocessed B-scans (a) and (c). The results show the detected hyperbola in red (Figures 6 (b) and (d)). Especially, Figure 6 (d) illustrates the detection result of the B-scan shown in Figure 4 (a) with five pipes.

In order to assess the detection rate of our method, both databases have been divided into three parts : a learning database which contains $60 \%$ of the whole database to learn the model, a cross-validation database which allows the best parameter selection and a test database to assess the detection performances with respectively $20 \%$ of the whole database. Four metrics (Eq:12, 13, 14, 15) are also applied.

The precision $(P)$ represents the good classification rate among positive predictions. The recall $(R)$ represents the good classification rate when the class in input is positive.

\#TruePositive $=\sum_{i=1}^{m} 1\left\{\right.$ prediction $_{i}=y_{i} \wedge$ prediction $\left._{i}=1\right\}$

$\#$ FalsePositive $=\sum_{i=1}^{m} 1\left\{\right.$ prediction $_{i} \neq y_{i} \wedge$ prediction $\left._{i}=1\right\}$

$\#$ FalseNegative $=\sum_{i=1}^{m} 1$ prediction $_{i} \neq y_{i} \wedge$ prediction $\left._{i}=0\right\}$

with $m$, the number of elements in the database, prediction the result of the algorithm for the $\mathrm{i}^{\text {th }}$ database element and $y_{i}$, the true value for the $\mathrm{i}^{\text {th }}$ element, we measure :

$$
\begin{gathered}
P=\frac{\# \text { TruePositive }}{\text { \#TruePositive }+\# \text { FalsePositive }} \\
R=\frac{\# \text { TruePositive }}{\# \text { TruePositive }+\# \text { FalseNegative }} \\
\text { F-Score }=2 \times \frac{P . R}{P+R}
\end{gathered}
$$

The good detection rate is also used which represents the good prediction percent regardless the class in input.

$$
\text { Rate } \left.=\frac{1}{m} \times \sum_{i=1}^{m} 1 \text { prediction }_{i}=y_{i}\right\}
$$

We compare our method with different supervised learning algorithms coupled with different features to learn. Firstly, a database composed of hyperbola patches and background patches have been learnt. Different descriptor algorithms have also been computed to extract features of this database, Histogram of Gradient (HOG) and the Canny Edge detector algorithms. Table II shows the results of the learning process on a test set. We noticed from these results that our method outperforms the detection results from hyperbola patches. 


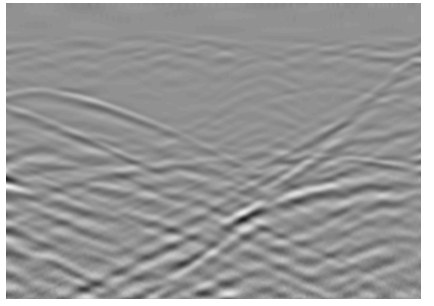

(a) Radar preprocessed 1

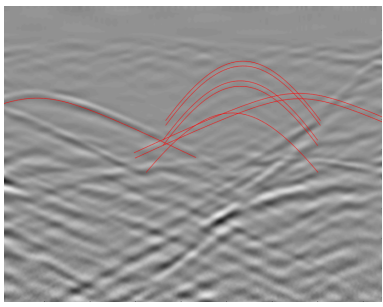

(b) Results 1

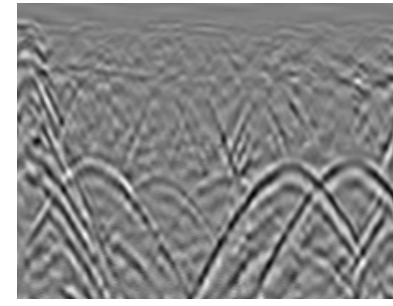

(c) Radar preprocessed 2

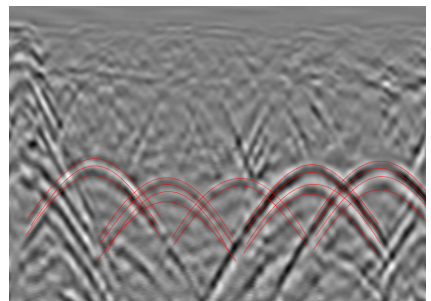

(d) Results 2

Figure 6: Automatic hyperbola localization results on two preprocessed B-scan (a) and (b) and their shape estimation in red (b) and (d)

\begin{tabular}{|l|l||c|c|c|c|}
\hline Feature & Kernel & Precision & Recall & F-Score & Rate \\
\hline \multirow{2}{*}{ Raw } & Linear & 57.9 & 53.5 & 55.6 & 55.8 \\
& RBF & 80.2 & 80.5 & 80.3 & 79.6 \\
\hline \multirow{2}{*}{ HOG } & Linear & 74.1 & 69.3 & 71.6 & 71.6 \\
& RBF & 78.1 & 80.3 & 79.2 & 78.2 \\
\hline \multirow{2}{*}{ Canny } & Linear & 58.2 & 55.7 & 56.9 & 56.4 \\
& RBF & 71.0 & 71.0 & 71.0 & 70 \\
\hline
\end{tabular}

Table I: Results (\%) of the model learning with SVM algorithm from the test hyperbola database

\begin{tabular}{|l|l||c|c|c|c|}
\hline Feature & Kernel & Precision & Recall & F-Score & Rate \\
\hline \multirow{2}{*}{ Coef. } & Linear & 66.7 & 75.5 & 70.9 & 69.2 \\
& RBF & $\mathbf{9 6 . 5}$ & $\mathbf{9 3 . 3}$ & $\mathbf{9 4 . 9}$ & $\mathbf{9 5 . 0}$ \\
\hline \multirow{2}{*}{ Blobs } & Linear & 55.0 & 59.0 & 56.9 & 54.6 \\
& RBF & $\mathbf{9 6 . 7}$ & $\mathbf{9 5 . 9}$ & $\mathbf{9 6 . 3}$ & $\mathbf{9 6 . 3}$ \\
\hline
\end{tabular}

Table II: Results (\%) of the model learning with SVM algorithm from the test coefficient and blob database

The use of hyperbola patches can be problematic. These patches may be too specific and do not cover all the possibilities. Indeed an hyperbola can be partially overlapped by clutter, distorted, polluted by noise or several hyperbolas may overlap each other. Moreover, hyperbola detection from patches does not appear to be the best solution. Several patch dimensions have to be considered. If the panel size is too restrictive the detection result will be bad. On one hand if patches size is too large, most of the clutter will be present and will distort the detection. On the other hand if it is not large enough to cover the whole hyperbola, it will risk being classified as clutter. Here the detection from the correlation between an hyperbola dictionary and B-scan reduces the impact of the background on the data representing the presence of the hyperbola (the positive class) and use a unique patch size.

\section{CONCLUSION}

In this work, we proposed a method to automatically detect hyperbolas in B-scan and thus to localize pipes in the underground. For this purpose, we chose to use a dictionary of simple theoretical measures of pipes coupled with a supervised learning scheme. For reasons of robustness and computation speed, two models have been learnt.

The first one uses the coefficient vectors obtained with the correlation between a B-scan and the dictionary as feature in input. The second one look for a blob at the kept positions by the first model for the atom with the highest correlation value. Especially Support Vector Machine algorithm has been chosen to learn both models. The results show a good detection rate of $95 \%$ for the coefficient vector model and upper than $96 \%$ for blob patch model.

In future works, complementary studies about the blob enhancement and on the dictionary should be done. A more focused study on supervised learning algorithm should also be undertaken.

\section{ACKNOWLEDGMENT}

The authors would like to thank BPI France and Région Île-de-France which have supported this work within the G4M project (http://g4m.fr).

\section{REFERENCES}

[1] M. de l'Écologie du Développement durable et de l'Énergie, "Inventaires 2013 des accidents domestiques," http://www.developpementdurable.gouv.fr/IMG/pdf/ inventaire_2013_web-2.pdf, [online : 2015-09-14].

[2] H. M. Jol, in Ground Penetrating Radar Theory and Applications. Amsterdam: Elsevier, 2009.

[3] C. Maas and J. Schmalzl, "Using pattern recognition to automatically localize reflection hyperbolas in data from ground penetrating radar," Computers and Geosciences, vol. 58, pp. 116-125, Août 2013.

[4] S. Birkenfeld, "Automatic detection of reflexion hyperbolas in GPR data with neural networks," in World Automation Congress. Kobe: IEEE, Septembre 2010, pp. 1-6.

[5] W. Al-Nuaimy, Y. Huang, M. Nakhkash, M. Fang, V. Nguyen, and A. Eriksen, "Automatic detection utilities and solid objects with GPR using neural networks and pattern recognition," Journal of Applied Geophysics, vol. 43, no. 2-4, pp. $157-165,2000$.

[6] W. Shao, A. Bouzerdoum, and S. L. Phung, "Sparse representation of GPR traces with application to signal classification," IEEE TGRS, vol. 51, no. 7, pp. 3922-3930, Juillet 2013.

[7] R. Zhang and T. J. Ulrych, "Physical wavelet frame denoising," Geophysics, vol. 68, no. 1, pp. 225-231, 2003.

[8] G. Terrasse, J. M. Nicolas, E. Trouvé, and E. Drouet, "Application of the curvelet transform for pipe detection in GPR images," in IGARSS, Milano, Italy, Jul. 2015.

[9] C. J. C. Burges, "A tutorial on support vector machines for pattern recognition," Data Min. Knowl. Discov., vol. 2, no. 2, pp. 121-167, Jun. 1998. [Online]. Available: http://dx.doi.org/10.1023/A:1009715923555

[10] "G4M," www.g4m.fr. 